\title{
ANEMIA E TRANSTORNO DO ESPECTRO AUTISTA: REVISÃO DE PRONTUÁRIOS
}

Kamila Castro

Programa de Pós-Graduação em Saúde da Criança e do Adolescente, Universidade Federal do Rio Grande do Sul, Hospital de Clínicas/Universidade Federal do Rio Grande do Sul, kamilacastro@hotmail.com.br Josemar Marchezan

Programa de Pós-Graduação em Saúde da Criança e do Adolescente, Universidade Federal do Rio Grande do Sul, j.marchezan@htomail.com Ingrid Schweigert Perry Programa de Pós-Graduação em Saúde Coletiva, Universidade do Extremo Sul Catarinense, atputp@gmail.com

\section{Rudimar dos Santos Riesgo}

Programa de Pós-Graduação em Saúde da Criança e do Adolescente, Universidade Federal do Rio Grande do Sul, rriesgo@hcpa.edu.br

\section{RESUMO}

Dados populacionais nacionais indicam prevalência de anemia de $20,9 \%$ e de $24,1 \%$ em crianças menores de cinco e dois anos de idade, respectivamente. Na regiáo sul, a prevalência de anemia aproxima-se dos dados nacionais $(21,5 \%)$. Estudos recentes apontam que a deficiência de ferro e anemia ferro- 
priva são mais comuns em crianças com atraso no desenvolvimento global e/ou transtorno do espectro autista (TEA) do que na população em geral. O ferro é um micronutriente crucial para o neurodesenvolvimento, contribuindo para a produção de neurotransmissores, mielinização e função imune, com considerável importância no desenvolvimento cognitivo, comportamental e motor. Diante disso, este estudo procurou investigar a prevalência de anemia em crianças e adolescentes com TEA, entre 3 e 18 anos, de ambos os sexos, acompanhados em ambulatório de neuropediatria, em um hospital público no sul do Brasil. Foi feita uma revisão retrospectiva de parâmetros hematimétricos em prontuários. Além disso, foram extraídos itens relativos à seletividade e/ou à restrição alimentar. Um total de 118 prontuários foram revisados quanto aos parâmetros hematimétricos (ferritina sérica, ferro, hemoglobina, hematócrito, volume corpuscular médio e grau de anisocitose). Ao total, 28,0\% dos pacientes apresentavam anemia, de acordo com níveis de hemoglobina. Nestes pacientes, 21,5\% tinham algum tipo de seletividade alimentar. Ao tratar a anemia classificada por meio da deficiência de ferro (ferritina abaixo de $12 \mathrm{mg} / \mathrm{L}$ para as crianças menores de cinco anos, e abaixo $15 \mathrm{de} \mu \mathrm{g} / \mathrm{L}$ para maiores de cinco anos), verificou-se que 19 pacientes apresentaram resultado positivo para anemia. A elevada frequência de adolescentes e crianças com TEA apresentando anemia sugere que estes parâmetros devem ser monitorados como parte de uma investigação de rotina no TEA, considerando seus efeitos deletérios sobre aspectos cognitivos e demais implicaçóes da anemia. Aspectos, como, por exemplo, seletividade alimentar/comportamento alimentar no TEA e uso crônico de certas medicações, poderiam constituir fatores sobrepostos para o desenvolvimento da anemia nestes indivíduos, sugerindo igualmente necessidade de vigilância.

Palavras-chave: Transtorno do Espectro Autista; Parâmetros Hematimétricos; Deficiência de Ferro; Anemia. 